\title{
Cellular and Humoral Responses following Minimally Invasive Surgery: Role of Reactive Oxygen Species
}

\author{
H Anne Leaver, Dino Rotondo ${ }^{\S}$, William S Walker. \\ Cell Biology Laboratory, Blood Transfusion, Clinical Neurosciences, University of \\ Edinburgh \& Cardiothoracic Surgery Department, Edinburgh Royal Infirmary \\ Edinburgh UK, §SIPBS, University of Strathclyde, Glasgow
}

Correspondence to: Dr HA Leaver, Cell Biology R\&D SNBTS, 21, Ellen's Glen Road, Edinburgh EH17 7QT, UK

Tel/Fax:+44 1315366792

Email: hanne.leaver@gmail.com

Running Title: Role of Reactive Oxygen Species in Minimally Invasive Surgery 


\begin{abstract}
Surgery is associated with release of local and systemic mediators which influence vascular and tissue homeostasis. The post-surgical release of acute phase reactants, particularly IL-6 and CRP, is reduced in minimally invasive surgery, compared with conventional surgery. Additionally, there is emerging evidence that leukocytes and Reactive Oxygen Species (ROS) are affected differentially by minimally invasive surgery.
\end{abstract}

ROS and mediators of oxidative stress influence vascular endothelium, organ perfusion and angiogenesis, and may be critical determinants of survival and outcome. ROS also influence cell proliferation and cell death and have been implicated in neoplastic signalling. Advances in ROS detection and oxidative stress signalling have shown cellular changes affected by minimally invasive surgery. It has been observed that the invasiveness of surgery is proportional to changes in phagocyte and lymphocyte ROS. A potential role for ROS signalling in the immune system is suggested by evidence of closer ROS/leukocyte correlation in patients undergoing minimally invasive surgery. At lower ROS levels, phagocyte and lymphocyte ROS following minimally invasive surgery implicate a homeostatic response with closer leukocyte/ROS correlations, whereas higher ROS in open surgery and greater leukocyte depletion indicates more cytotoxic signalling.

Interactions between humoral and cellular elements suggest that an integrated systems approach, using individual patient responses, should be used to analyse the response to surgical trauma and oxidative stress. These studies are being used to investigate ROS as biomarkers of the effects of surgery on vascular and pulmonary reactivity. ROS signalling may provide insights into post surgical trauma and its underlying pathology.

Keywords: Mediators, Minimally Invasive Surgery, Lobectomy, ROS 


\section{Introduction}

There is increasing evidence for the advantages of minimally invasive surgery in a variety of pathologies $[1,2]$. However, there is surprisingly little quantitative analysis of molecular and cellular processes differentially affected by minimally invasive surgery. This is partly because multi-factorial processes are involved. These include less blood loss and transfusion, lower anaesthetic use, and reduced tissue trauma in minimally invasive surgery [3]. In this review, these diverse factors and networks will be discussed in terms of cellular and humoral mediators and Reactive Oxygen Species (ROS) signalling as a regulatory process in surgical stress.

The networks affected by surgical processes include immune, inflammatory, stress signalling and vascular systems. Circulating biomarkers of these networks provide evidence of mediators and cellular responses differentially affected by minimally invasive surgery [4-7]. There have been advances in the characterisation of mediators released during surgery, including eicosanoids, anaphylactic mediators [8-14] and factors affecting pulmonary oncogenesis [15-18]. An early response to surgery is the release, within minutes to hours, of humoral mediators including acute phase proteins, cytokines, ROS [19-21], lipid mediators, adrenocorticoids and catecholamines. These mediators influence vascular permeability, tissue perfusion and metabolic breakdown processes, known as catabolism [22-24]. Although some of these responses result in homeostasis, the catabolic state may be serious or fatal, especially in the immediate post surgical period. Over a longer period, hours to days after surgery, changes in humoral immune mediators are associated with altered leukocyte distribution and activity [7]. There is evidence of ROS involvement in immune responses, where lymphocyte ROS are associated with proliferative signalling at low concentrations and inhibition of lymphocyte activity at higher concentrations. Humoral immune mediators (including ROS) influence the immune system via host specific antibody production [17] and act on effector cells such as mononuclear and polymorphonuclear phagocytes and natural killer (NK) cells $[4,25]$. The cellular arm of the immune system therefore responds to circulating and local mediators, and produces ROS and other mediators in response to surgical trauma. It will be proposed that a regulatory response to surgical trauma lies in the reticulo-endothelial system, where ROS associated endothelial damage may result in multi organ failure, or influence tumour development, angiogenesis and metastasis [26- 
28]. The effects of minimally invasive surgery on various endpoints must therefore be considered. In addition to survival, effects on immunity, oncogenic processes and vascular and tissue metabolism must be determined.

This review will analyse humoral and cellular mediators differentially affected by minimally invasive surgery compared with open surgery (Table 1), with specific focus on early triggering mediators detected during the surgery of pulmonary neoplasms. The impact of surgical trauma on circulating immune cells will be discussed in relation to ROS effects on innate and acquired immunity and the vasculature. Recent evidence of interactions between immunological and humoral mediators, indicating connectivity between humoral and cellular mediators of surgical stress will be presented.

Accumulating evidence, suggesting that minimally invasive techniques reduce specific stress responses, leading to different cellular fates and reduced cytotoxicity, will be reviewed. Correlations between ROS activity and leukocyte distribution also suggest that further attention should be paid to metabolic and oxidative activities at cellular and systemic levels.

Table 1. Circulating Cellular and Humoral Mediators of Surgical Stress affected differentially by Minimally Invasive Surgery. The response to open surgery and minimally invasive surgery (brackets) is indicated by + (increase) or - (decrease). $\operatorname{IgG}$, IgM, IgA (Immunoglobulins G, M, A); IGFBP-3 (insulin growth factor binding protein$3)$;

\begin{tabular}{|l|c|c|l|}
\hline Mediator/ Biomarker & Response & Peak change & Ref \\
Interleukin-6 & $+++(++/-)$ & $4-24 \mathrm{~h}$ & {$[4,19]$} \\
C-reactive protein & $+++(++)$ & $48 \mathrm{~h}$ & {$[19,2]$} \\
Serum amyloid-A & $+++(++)$ & $48 \mathrm{~h}$ & {$[29]$} \\
Phagocyte numbers, activity & $++(+)$ & $24 \mathrm{~h}$ & {$[6,20]$} \\
ROS & $++(+)$ & $48 \mathrm{~h}$ & {$[12,20]$} \\
Lymphocytes (T,B, CD4/8, NK) & $--(+)$ & $48 \mathrm{~h}$ & {$[4,7,29]$} \\
IgG, IgM and IgA & $--(--)$ & $48 \mathrm{~h}$ & {$[4,17]$} \\
Immunokine (IGFBP-3, MMP-9) & $--(-),++(+)$ & $72 \mathrm{~h}$ & {$[29]$} \\
\hline
\end{tabular}




\section{Circulating Cellular and Humoral Mediators of Surgical Stress affected differentially by Minimally Invasive Surgery}

It is well established that a primary response to surgical stress is the acute phase response, in which acute phase proteins, including C-Reactive Protein (CRP), serum amyloid A, the acute phase cytokine, Interleukin-6 (IL-6) and complement are rapidly released (Table 1 and Fig 1). This response is distinct from the elevation of acute phase reactants associated with infectious complications, and depends on tissue type, as well as the invasiveness of surgery. Acute phase and cytokine changes associated with minimally invasive surgery are discussed further below.

Other changes in small molecular weight metabolites and serum metabolites have also been detected in minimally invasive surgery, compared with conventional surgery, such as urea and haemoglobin. However, these metabolites are more closely linked to renal function and haemodilution, which are less affected by minimally invasive surgery. Local pH and perfusion (pO2, pCO2) may also be linked to blood loss [30]. Many other metabolites affected by minimally invasive surgery also tend to be localized in excised tissue and vasculature. Intermediary metabolites associated with the stress response include $\alpha$-ketoglutarate/glutamate/glutathione, which influence ROS production and bioactivity, and succinate and oxaloacetate in the citric acid cycle and pyruvate/acetate in gluconeogenesis which are a target of ROS activity [31]. However, a wide range of metabolites including lipoprotein, glucose, lactate, pyruvate, citrate, formate, acetate and amino acids are also elevated in tumour tissue [32]. Therefore, it is essential to monitor presurgical levels of these metabolites and to determine changes in individual patients after surgery.

Another group of metabolites associated with the invasiveness of surgery are linked to vascular endothelial function. Thus, in the brain, glutamate may report on endothelial cell function and the permeability of the blood brain barrier following surgery [33], and there is preliminary evidence that interstitial pyruvate and amino acids may link to consciousness in a rabbit model of subarachnoid haemorrhage [34]. Soluble adhesion molecules may also report on the extent of surgical invasiveness. Other circulating factors recognised to be differentially influenced by minimally invasive surgery include immunoglobulins and the related T and B cells, although these factors 
are also affected by blood loss. Neurendocrine factors also affect the stress response in surgical patients [35] but have not been analysed in prospective randomised trials of minimally invasive surgery. These factors may also reflect anaesthesia levels, which are often lower in minimally invasive surgery.

\section{Acute Phase Response, Cytokines and ROS following Minimally Invasive Surgery}

During the acute phase response to surgical stress. acute phase proteins, the acute phase cytokine, Interleukin-6 (IL-6) and complement are rapidly released (Fig 1). This response depends on the invasiveness of surgery. Following over twenty years of cytokine analysis, a specific sequence of cytokine changes have been linked to surgery, and there is recent evidence that these affect subsequent ROS and microvascular responses.

The cytokine profile associated with surgical trauma (transiently high IL-6, low tumour necrosis factor- $\alpha$;NF- $\alpha$ ) is affected differentially by minimally invasive techniques. A characteristic of minimally invasive surgery is attenuation of post surgical CRP and IL-6 changes, compared with conventional surgery $[3,36]$. These cytokine signals are closely linked to ROS, eicosanoid and lipoxygenase products, which have specific effects on pulmonary inflammation, oncogenesis and angiogenesis [8, 12, 37, 38]. Associated procedures and processes such as transfusion and haemorrhage also modulate the cytokine profile associated with trauma [5]. IL-6 and activated complement are released from tissue during surgery, and pulmonary mRNA expression of IL-6 and proinflammatory cytokines increase following surgery [21]. CRP and cytokines including IL-6 are also released during muscle injury [39]. The effects of acute phase reactants and cytokines associated with surgical stress on oxidative metabolism are complex, both facilitating ROS release in the early phase and down-regulating ROS damage in the later phase of the stress response. IL-6 is important in initiating inflammatory type reactions, while CRP binds damaged tissue, nuclear antigens and certain pathogens, assisting in their clearance. CRP acts in the innate immune system. Like IgG, CRP activates complement and binds Fc receptors, leading to an amplification of pro-inflammatory cytokine generation. Complement Factor H was recently shown to protect against oxidative stress by binding lipoxidation products [40]. CRP also recognizes altered self 
and foreign molecules, providing an early defense, and activating adaptive immunity [41].

While the cytokine profile for different surgical targets varies, generally the minimally invasive route is associated with attenuation of stress-related cytokine signalling. Thus, in a study of minimally invasive and open surgery for rectal cancer, IL-2 decreased after surgery in both groups. However, pro-inflammatory IL-6 decreased 1 and 5 days after minimally invasive surgery, but increased after open surgery, while no change in TNF was detected [4]. Characteristic cytokine changes after pulmonary lobectomy included elevated IL-6, with substantially greater increases 48 hours after open surgery [19]. IL-6 regulates the release of factors affecting tissue oxidative metabolism and the tissue microenvironment, which have been reported to be differentially affected by minimally invasive surgery. Insulin growth factor binding protein (IGFBP-3), which is inhibited by IL-6, increased, and matrix metalloproteinase (MMP-9), which is stimulated by IL-6, decreased in minimally invasive, compared to open surgery [29]. Matrix metalloproteases and oxidative metabolism are associated with local ROS formation (see Mediators of Oxidative Stress, below) and these influence immune surveillance and tumour recurrence. Effects of minimally invasive surgery on immunoglobulins and leucocyte ROS have been observed [20] and will also be discussed.

Fig 1. Interactions of ROS, Humoral and Cellular Mediators of Pulmonary Surgery. Mediators differentially affected by minimally invasive surgery are shown, together with products of lipid oxidation, prostaglandins (PGs) and lipoxygenase products (LO). Differential effects of moderate (green) and high (red) ROS levels on cells of the vasculature and lung parenchyma are shown. Humoral mediators and associated ROS signalling may link surgical stress and pathophysiological responses. Thus, high ROS concentrations can rapidly disrupt vascular endothelial and endothelial cell function and influence tumour associated angiogenesis. At lower ROS concentrations, however, homeostatic responses have been detected. It is proposed that lower ROS levels associated with minimal access surgery are involved in homeostatic change in immune and reticuloendothelial responses. At higher levels, ROS may exert cytotoxicity towards residual tumour cells. 


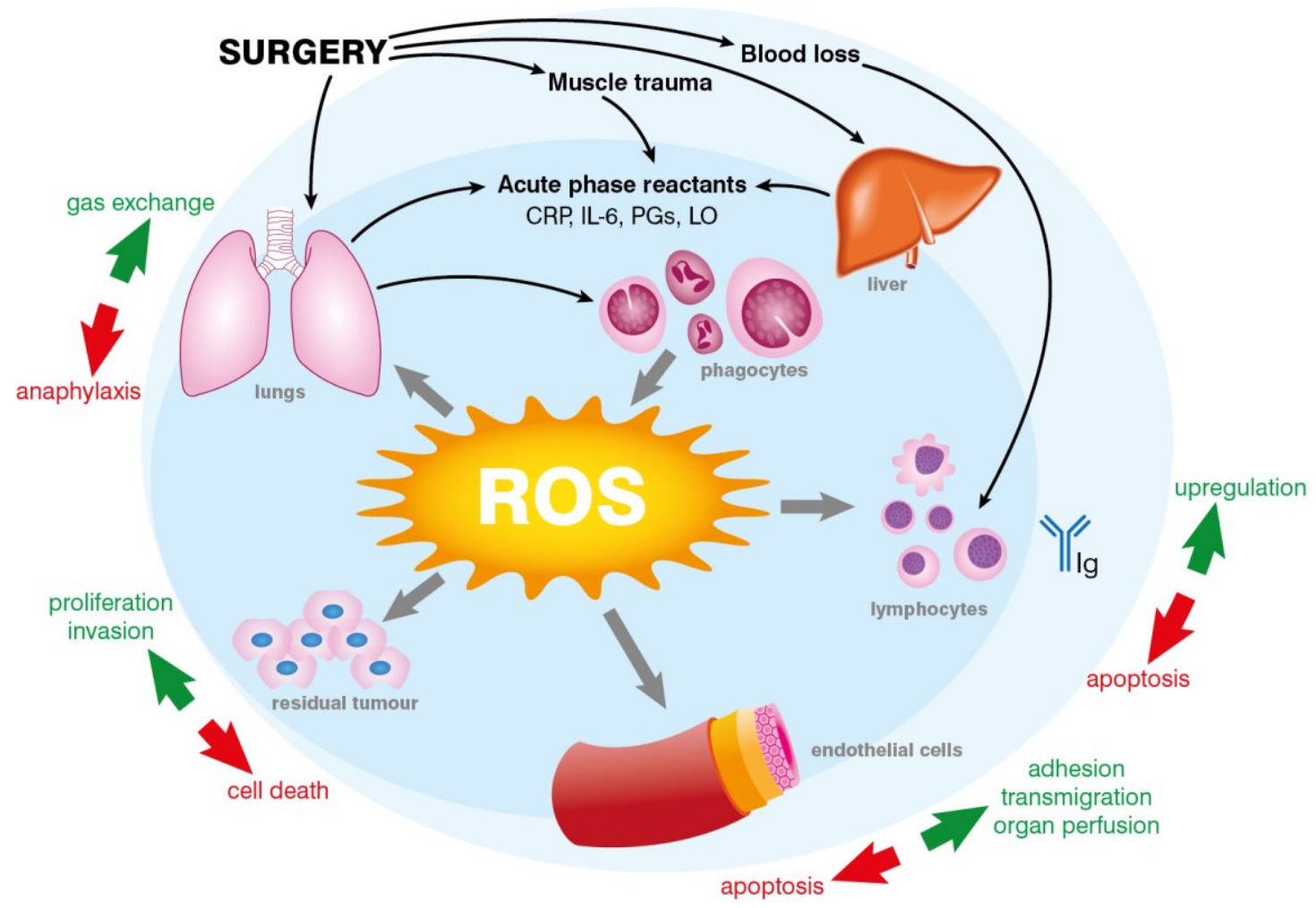

Surgery and Oxidative Stress

Recent advances in ROS analysis have improved our understanding of oxidative stress in patients undergoing surgery. Nanotechnology and microarrays to detect localized cytokine, immunokine and lipid mediators in cells and tissues have been linked to probes sensitive to ROS and redox activity [21]. These approaches require rigorous standardization of cellular and patient responses. However, they can be used to detect rapid signalling and metabolic changes in blood vessels and tissues in vivo. The concept of ROS as signalling elements requires different approaches to analysis, due to the distinct character of ROS, compared with other humoral signals such as cytokines and acute phase proteins. ROS are able to cross membranes and therefore their targets may be intracellular and transcellular (see Cellular Mediators of Oxidative Stress section below). However, ROS are also relatively short range, localized mediators, due to their rapid turnover and antioxidant activity (Table 2).

Normal oxidative metabolism leads to limited ROS generation which is below the antioxidant capacity of the cell. Both ROS generation and antioxidant activity vary 
between cell types, however, the balance between ROS production and consumption, and the localisation of these processes is crucial in determining homeostatic and cytotoxic responses (see Fig 1). In particular, high levels of superoxide anion (O2-) and its dismutation product, hydrogen peroxide, may lead to oxidative stress. Additionally, oxidative stress may arise through specialized enzymes of the reticuloendothelial system, such as NADPH oxidase, nitric oxide (NO) synthetase, cyclooxygenase, and lipoxidase, which produce hydrogen peroxide, superoxide, hydroperoxide, NO, peroxynitrite and lipid peroxides (Table 2). Oxidative stress is associated with local and systemic release of ROS, cytokines, lipid and stress mediators. Thus, the extent and characteristics of oxidative stress depends on micro-environmental factors and cellular ROS producing enzymes (which are highly active in phagocytes in the innate immune system). There is currently much research into cellular mediators of ROS action. These mediators are diverse, they depend on cellular redox activity and factors induced by surgical trauma [42], and vary between cells and tissues [43, 44]. This is particularly relevant to pulmonary surgery, where oxygen concentrations are high. Pulmonary ROS are closely regulated, and specific pathways control pulmonary endothelium, whose vasoactivity is selectively sensitive to lipoxygenase mediators and oxygen tension [15, 16, 45]. Cells derived from non small lung cancer are also highly sensitive to ROS [8, 46].

ROS formed during oxidative stress are transiently produced, but can have profound effects on cell and tissue metabolism and organ fate [9, 16, 24, 34]. ROS change cellular responses through diverse mechanisms. At low levels, ROS may act as signalling molecules, whereas higher localized ROS concentrations may damage organelles, particularly mitochondria. Oxidative damage and associated mitochondrial dysfunction may elicit energy depletion, accumulation of cytotoxic mediators and cell death $[47,48]$. Thus, while low ROS levels often stimulate physiological growth and activity, higher levels may lead to oxidative stress and pathological responses in vascular and pulmonary systems [23]. The interface between stress adaptation and cell death is important in disease pathogenesis. Autophagy, one of the main catabolic pathways, links these processes and has also been postulated to play a role in hypoxia and tumour development $[49,50]$. 
Table 2 ROS formation in Human Cells and Tissues.

\begin{tabular}{|l|l|}
\hline $\begin{array}{l}\text { Mediators of Oxidative Stress } \\
\text { Superoxide [16, 43] } \\
\text { Hydrogen peroxide [51] } \\
\text { Hydroperoxide } \\
\text { Peroxynitrite } \\
\text { Lipid peroxides [8, 45] }\end{array}$ & $\begin{array}{l}\text { Metabolic Factors } \\
\text { Oxygen } \\
\text { Glucose } \\
\text { ATP } \\
\text { Redox active iron(haemolysis) [52, 53] } \\
\text { Polyunsaturated fatty acids [44, 47, 48] } \\
\text { Phagocytes [51] }\end{array}$ \\
\begin{tabular}{|l|l|} 
ROS production \\
Mitochondrial respiratory chain[37, 41] \\
NADPH oxidase (phagocytes, endothelial cells, erythrocytes) [24, 27] \\
Nitric oxide synthetase (eNOS, nNOS, iNOS) [27] \\
Xanthine oxidase \\
Monoamine oxidase \\
Lipoperoxidases [43-45]
\end{tabular} \\
\hline $\begin{array}{l}\text { Antioxidants } \\
\text { Reduced glutathione (GSH) } \\
\text { Vitamin C, E }\end{array}$ \\
\hline
\end{tabular}

\section{Phagocyte ROS in Minimally Invasive Surgery}

There is increasing evidence that phagocyte ROS are up-regulated by surgery, and that minimally invasive techniques modulate this release. Recent evidence suggests that ROS production following minimally invasive surgery falls close to the transition point between homeostatic and cytotoxic responses. An effect of minimally invasive thoracic surgery (VATS) has been reported on phagocyte ROS [6, 20]. Postsurgical ROS increased in monocytes and neutrophils, using an assay which indicated ROS/ mortality associations in patients with sepsis [54]. Open surgery elicited twice as much neutrophil ROS as minimally invasive surgery two days after pulmonary surgery [20]. In order to analyse the correlation between ROS and leukocyte mobilisation, blood leukocyte counts and their oxidative activity were compared in individual patients. NK cells and phagocyte 
ROS showed different changes after minimally invasive and open surgery (Fig 3A). The higher ROS in open surgery patients showed negative correlations, when ROS and lymphocyte numbers were compared. This may reflect leukocyte cytotoxicity and tissue and reticulo-endothelial uptake, leading to loss of circulating lymphocytes. In contrast, positive correlations in minimally invasive surgery patients indicated tighter coupling between leukocyte oxidation and lymphocyte numbers.

Thus, moderate ROS production in minimal access surgery may exert a homeostatic function, where antioxidant defences are able to limit ROS associated cytotoxicity (Fig 1). A homeostatic link between immune factors and phagocyte ROS has been reported in immunodeficient patients, where IgG inhibited monocyte ROS and stimulated CD8 lymphocyte count [55]. However, there is evidence that activated phagocytes may also inhibit lymphocyte reactivity via ROS: At higher ROS concentrations, activated human blood monocytes and neutrophils suppressed lymphocyte mitogenesis, suggesting a cytolethal suppressive mechanism. Suppression was prevented by catalase, implicating $\mathrm{H}_{2} \mathrm{O}_{2}$ as a mediator [51].

\section{Lymphocyte ROS in Minimally Invasive Surgery}

Minimally invasive surgery results in moderate immunosupression [3, 4, 7] and disturbances of immune function may predispose to increased tumour growth/recurrence. Decreases in circulating immunoglobulin and lymphocytes provide evidence of post surgical immunosuppression, which is reduced in minimally invasive surgery, Leaver et al 2014, Cellular Metabolomics, 2 (3) in press. T and B lymphocytes play key roles in post surgical infection. Changes in T and B leukocyte and NK cell numbers, and decreased $\mathrm{T}$ helper/suppressor cell ratios (CD4/8) were detected 7 days post- surgery and were lower in patients undergoing minimal access surgery, indicating a cellular immune system which was less affected by minimal access surgery [20, 36]. In lung cancer, NK cells play a cytotoxic role [56], and acquired immune responses are involved in tumour recognition [57] and killing. In rectal cancer, NK cells decreased postoperatively in laparoscopic and open surgery. [4]. Postoperative IgG and IgA decreases after open and closed heart surgery were more significant after open surgery, and reflected decreased antibacterial immunity and higher infection rates in open surgery [17]. Decreased serum IgA, IgG and IgM also occur following liver transplantation: rapid decreases on the first 
few days after surgery were followed by moderate increases [58]. In a prospective study of pulmonary lobectomy, IgG, IgA, and IgM decreases showed close correlations (Fig 2), suggesting similar depletion processes. Similar post-surgical changes were detected in laparoscopic cholecytstectomy [59].

Cellular changes differentially affected by minimally invasive surgery include leukocyte cell numbers [7] and their oxidative activity [20]. Until recently, lymphocyte ROS production received less attention than the antimicrobial role of ROS in innate immunity. However, there is evidence for ROS signalling in adaptive immunity. T lymphocyte activation, through specific antigen receptor, $\mathrm{T}$ cell receptor (TCR), is vital in regulating the immune response. Redox dependent $\mathrm{T}$ cell activation was detected during $\mathrm{T}$ cell signalling and gene expression, when TCR engagement induced rapid ROS production $[60,61]$.

There is also evidence of lymphocyte ROS signalling in inflammatory disease, as T lymphocyte ROS correlate with T cell mitogenic responsiveness [62]. Increased ROS signalling was associated with intravenous iron preparations in haemodialysis patients, which reduced lymphocyte survival [52]. Lymphocyte ROS increased 15\% following open surgery, but only $4 \%$ two days after minimal access surgery [20]. Lymphocyte ROS resembled phagocyte ROS, showing closer correlation in minimal access, compared with open surgery (Fig 3B). Thus, 48 hours after surgery in minimally invasive surgery patients, lymphocyte ROS correlated positively with lymphocyte numbers. However, in open surgery, there was negative correlation between higher ROS and lymphocyte numbers. These differences were transient, and were greater at two days after surgery. 


\section{A phagocyte ROS}

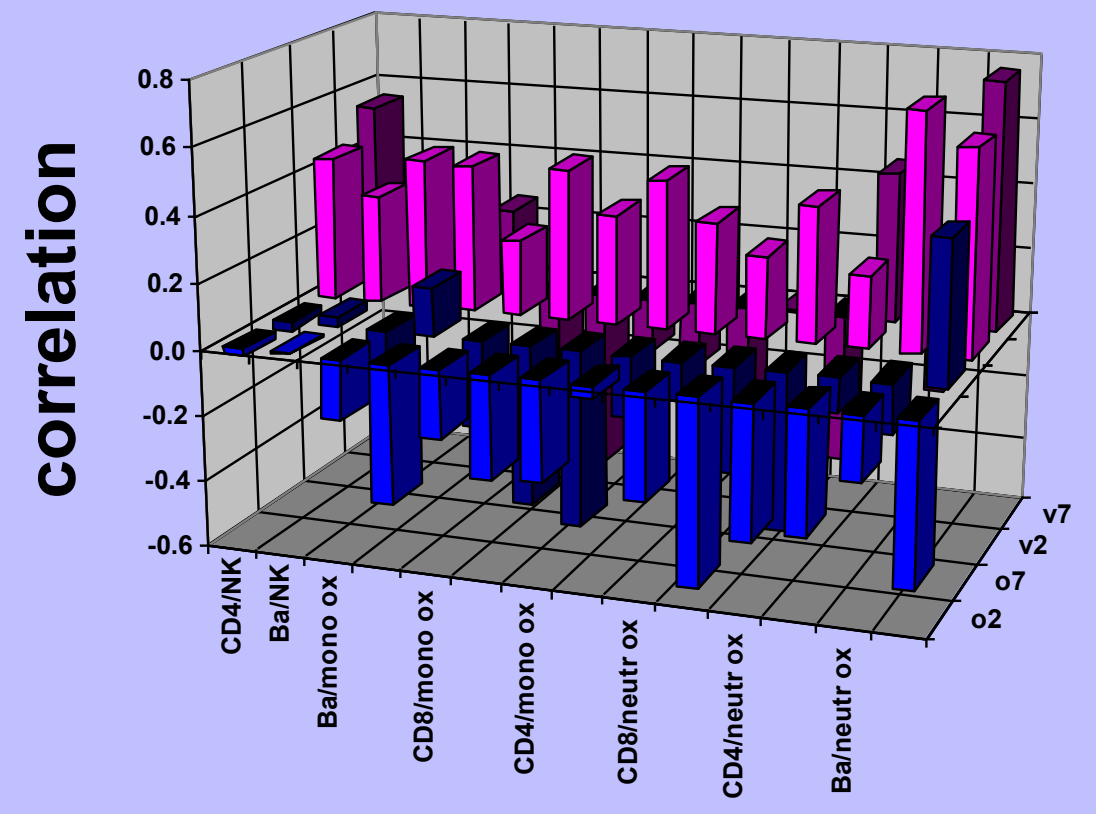

\section{B lymphocyte ROS}

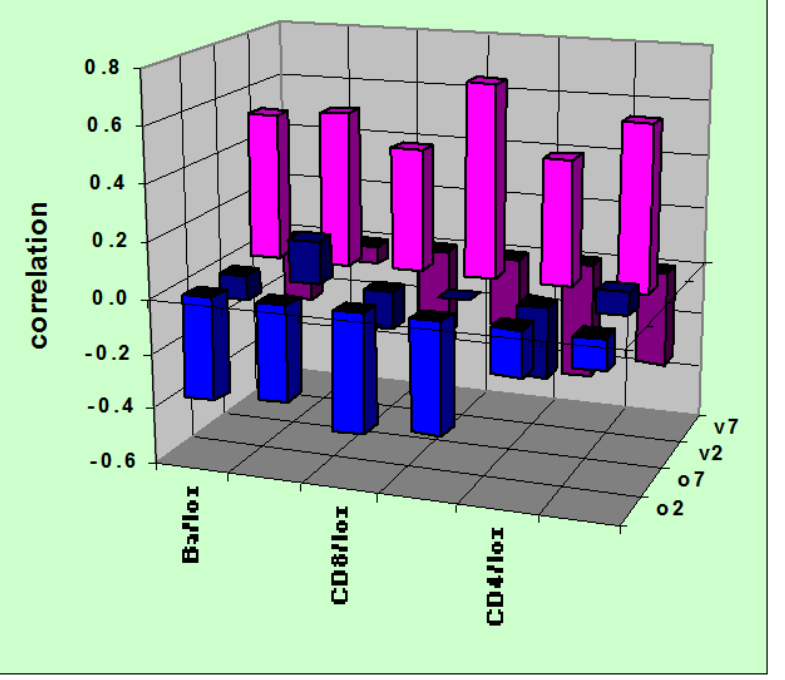

Fig 2. Cellular Immune Responses and ROS in Leukocytes of patients undergoing Open (o, blue bars) and Minimally Invasive (v, pink bars) Thoracotomy. Peripheral blood leukocytes and ROS in 41 patients undergoing pulmonary lobectomy were compared, leukocyte oxidative activity (ox) was analysed, using healthy blood donors as control. A) Correlation of post surgical change in absolute lymphocyte count (Ba, CD8a, CD4a) and absolute Natural Killer count (NKa), monocyte (mono ox) and neutrophil (neutr ox) ROS were compared on 2 and 7 days post op for open surgery (o2, o7) and VATS (v2, v7) patients. B) Correlation of lymphocyte and lymphocyte ROS (lox) in same patients. 


\section{Cellular Mediators of Oxidative Stress}

The importance of oxidative signalling in response to surgery is shown in the early genes up-regulated in leukocytes. Of the 17 up-regulated genes detected four hours after cardiac surgery, $53 \%$ were related to oxidative and metabolic stress signalling, while only $30 \%$ were linked to cytokine and chemokine responses [21]. In this section, some of the primary cellular targets of ROS will be discussed in relation to surgical stress and the networks affected by surgery.

A primary response factor sensitive to ROS is transcription factor NF- $\kappa \mathrm{B}$, which plays a major role in coordinating innate and adaptative immunity, cell proliferation, apoptosis

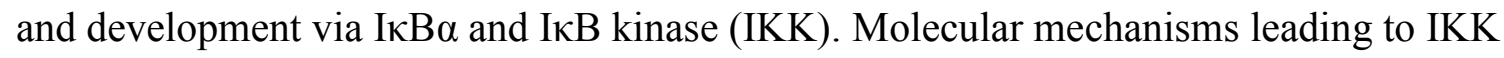
activation are cell specific, and various ROS including $\mathrm{HOCl}$, superoxide and reactive nitrogen species affect NF- $\kappa \mathrm{B}$. ROS are involved in NF- $\kappa \mathrm{B}$ activation by pro-inflammatory cytokines (TNF- $\alpha$, IL-1 $\beta$ ) [63-65]. Another key transcription factor in ROS signalling and tumour progression is hypoxia-inducible factor (HIF) [18, 66, 67]. Recent research suggests that HIF induction has serious consequences in diseases with a chronic inflammatory component, such as lung cancer $[15,68]$. It has also been shown that chronic inflammation is self-perpetuating, distorting the microenvironment and eliciting aberrant transcription factors. Both NF- $\kappa \mathrm{B}$ and HIF interact with lipid mediators and cytokine networks in response to ROS associated signals, and vascular and transformed cells are specific targets of this signalling [69].

ROS also influence endothelial cell function via Vascular Cell Adhesion Molecule-1 (VCAM-1), which, in concert with microenvironmental factors, regulates leukocyte migration from blood into tissues. Endothelial VCAM-1 expression is induced during inflammatory bowel disease, atherosclerosis, allograft rejection, infection and asthma. VCAM-1 signals are mediated by ROS and matrix metalloproteinases (signals required for endothelial cell shape change and leukocyte migration). Additionally, endothelial cell VCAM-1-activated signals are regulated by inflammatory cytokines [70 ]. A link between blood phagocyte activation, ROS and oxidative stress following reperfusion was 
suggested by upregulation of leukocyte adhesion and migration receptors following cardiac surgery [21] . Additionally, oxidative stress is increased following reventillation [71]. Further cellular influences, related to immunological mechanisms and ROS were detected in erythrocyte transfusions. Aged erythrocytes are associated with complications following cardiac surgery [72] and oxidative stress has been proposed to initiate RBC ageing processes [73]. A feedback link between ROS and the intrinsic immune system lies in signal inhibitory receptor on leukocytes-1 (SIRL-1). SIRL-1 ligation dampens Fc receptor-induced ROS production in primary human phagocytes [74].

High ROS concentrations can cause cell death in lung cancer cells, and there is increasing evidence that this is a primary pathway of tumour regression [46, 47]. Microenvironmental elements controlling tumour transformation, development and associated vascularisation are currently the focus of therapeutic development. Apoptosis of human lung epithelial cancer H460 cells, inhibited by ROS scavengers or antioxidant enzymes (glutathione peroxidase and superoxide dismutase), indicate a role for ROS, especially hydroperoxide and superoxide anion, via the mitochondrial death pathway, involving caspase-9 and down-regulation of mitochondrial Bcl-2 through peroxide-dependent proteasomal degradation [16]. However, ROS are also involved in tumour progression [75] and resistance to anti-proliferative signals e.g. increased human cardiac fibroblast resistance to oxidative stress associated with elevated FOXO3a, involving receptorinteracting kinase 1 and $\mathrm{TNF} \alpha$ [43]. ROS signalling elements are therefore being used to characterize tumour regression pathways and signals. The effects of the lower ROS levels associated with minimally invasive surgery, and whether localized ROS amplification at the site of surgery would be desirable requires further investigation.

\section{Direct and Indirect Approaches to ROS Analysis: Metabolomics and Cell Biology}

As described in Table 2, a range of metabolites and systems are involved in ROS production and bioactivity, and an informed metabolomic approach should ideally analyse multiple overlapping products and targets. This is particularly relevant in the clinical setting, where underlying vascular, metabolic, degenerative or oncogenic disease may affect ROS production and responses. 
ROS may be measured either directly, using spin trap methods or fluorescent substrates, or indirectly, in terms of ROS producing systems, or systems and metabolites affected by ROS. Some of the early cellular and mediator responses, such as stress kinases, stress factors such as HIF, matrix metalloproteases and apoptosis signals have already been described. A complimentary approach is to determine metabolic flux in the enzyme systems and organelles that produce and respond to ROS, such as NADPH oxidase, and mitochondria (membrane potential, permeability transition pore, cytochrome c release). Improved metabolic indicator analysis to determine respiratory control and redox status are widely used in toxicology and oncology to determine bioenergetic status, including ATP/ADP ratios, lipid oxidation products and metabolites increased by ROS. Intermediary metabolites which influence ROS production and bioactivity include $\alpha$ ketoglutarate/glutamate/glutathione, and targets of ROS include succinate and oxaloacetate in the citric acid cycle and pyruvate/acetate in gluconeogenesis [31]. Additionally, an important indicator of ROS bioactivity is antioxidant status, both intracellular and extracellular. Thus thiol group redox status and glutathione levels are often monitored, particularly in pharmaceutical and nutraceutical contexts, where many novel redox active compounds, often derived from traditional medicines, such as the flavinoids are showing promise. In summary, in the rapidly growing field of ROS characterization, some of the strongest evidence comes from multidisciplinary approaches, but metabolomics is at the forefront of advances in analysis of bioactivity and potential therapeutic advances.

\section{Conclusions}

Surgery elicits the release of an interconnected network of mediators, acting in patients whose vascular system may be compromised by underlying disease. In minimally invasive surgery, reduced acute phase responses have been observed, compared with conventional surgery. Post-surgical anergy is also a recognised effect in hours and days immediately following surgery, and differential effects of minimally invasive surgery have also been detected on circulating lymphocytes and secreted immunoglobulins (Table 1, Fig 2). 
Fig 3. Dendrograph indicating links between ROS and Humoral and Cellular Mediators following Surgery. This figure shows the correlation (red: negative, green: positive) between changes in factors reported to differ in A) Open surgery, and B) Minimally invasive (VATS) patients after surgery. Indicators of haemodilution: urea and blood loss (BL) $24 \mathrm{~h}$ after surgery showed negative correlation with Haemoglobin $(\mathrm{Hb})$, this correlation was similar in VATS patients. Also, correlations between IgG and IgA were similar in VATS and open surgery, 2 days after surgery. In contrast, there was a negative correlation between lymphocyte number/lymphocyte ROS 2 days after open surgery, but there was a positive correlation in VATS patients, supporting the hypothesis that higher ROS levels in open surgery were cytotoxic. Differences between VATS/open surgery were also detected in phagocyte monocyte (mROS) and neutrophil (nROS), compared with Complement (C3 and C4) 7 days after surgery. Greater negative correlations between complement $\mathrm{C} 3$ and $\mathrm{C} 4$ and phagocyte ROS were detected in the open surgery group.

\section{A Open Surgery}

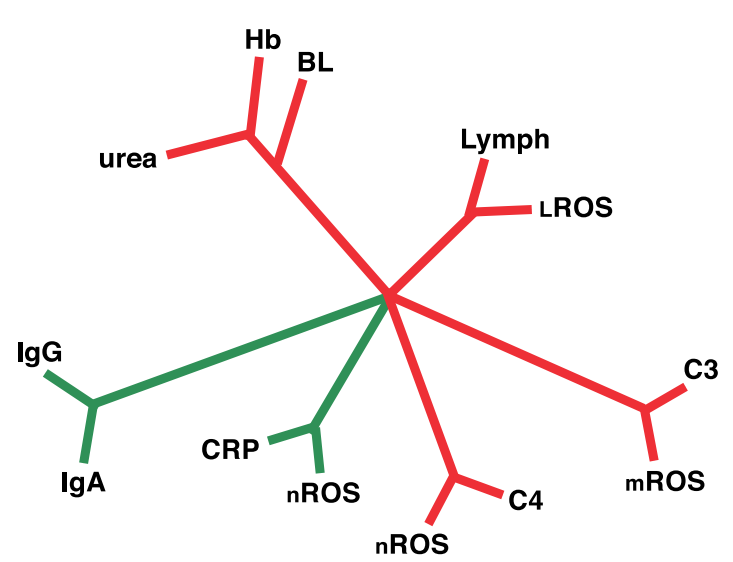

\section{B Minimally Invasive}

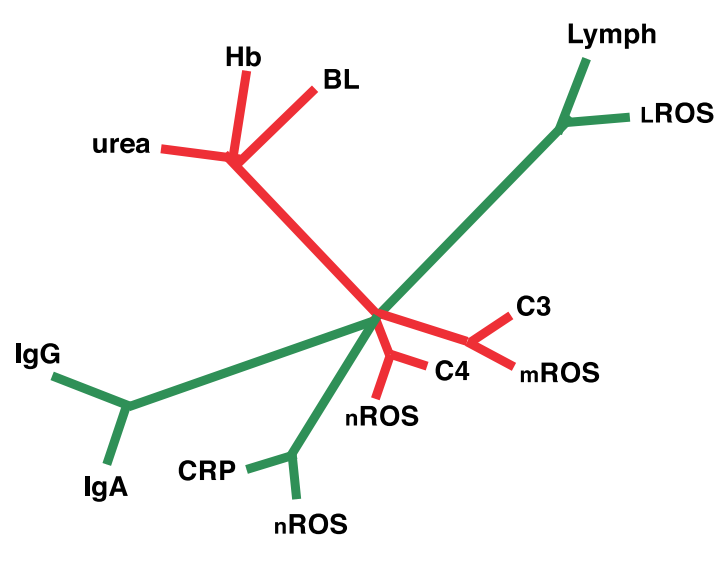

A factor that links humoral and cellular responses to surgical stress is oxidative metabolism in leukocytes and the vasculature. The extent of phagocyte and lymphocyte ROS production was also proportional to the degree of invasiveness of surgery. ROS research is a rapidly developing field, and more evidence is currently available from circulating ROS associated signals following surgery than ROS effects on target tissues. However, it is well established that leukocyte ROS have effects on vascular endothelium, organ perfusion and angiogenesis (Fig 1). Phagocyte and lymphocyte ROS also influence the immune system, with low concentrations stimulating mitogenesis and lymphocyte proliferation, and higher concentrations being inhibitory and cytotoxic. ROS associated interactions between systemic and cellular elements suggest that an integrated systems 
approach, involving individual patient responses, is important in analysing the effects of surgery on immunity and oncogenesis.

With their rapid release, ability to cross membranes, and sensitivity to microenvironmental and cell specific signals, ROS play a local signalling role, mediating cell and tissue homeostasis and damage responses. Future investigations of the impact of minimally invasive surgery should include rapidly turning over mediators of oxidative stress, their immunological activities (Figs 2 and 3 ) and vasoactivity in the immediate post surgical period. Additionally, pulmonary effects of these mediators must be considered. Finally, chronic influences are important in this patient group, where periods of hypoxia and vascular damage may lead to tissue damage or tumour development. It is important to analyse localized vascular and pulmonary changes, and new imaging techniques [76], together with microanalysis and probes of ROS and oxidative stress [23, 77] will provide insight into these processes.

\section{Acknowledgements}

We are grateful to Giuliana Rotondo for drawing the dendrograph. Conflicts of interest: none declared. This work was supported in part by grants from the Melville Trust for the Care and Cure of Cancer and Chest Heart and Stroke Scotland. 


\section{References}

[1] Ceppa, D.P.;Kosinski, A.S.;Berry, M.F.;Tong, B.C.;Harpole, D.H.;Mitchell, J.D.;D'Amico, T.A., Onaitis, M.W. Thoracoscopic lobectomy has increasing benefit in patients with poor pulmonary function: a Society of Thoracic Surgeons Database analysis. Ann. Surg. 2012, 256, 487-493.

[2] Flores, R.M.;Ihekweazu, U.N.;Rizk, N.;Dycoco, J.;Bains, M.S.;Downey, R.J.;Adusumilli, P.;Finley, D.J.;Huang, J.;Rusch, V.W.;Sarkaria, I., Park, B. Patterns of recurrence and incidence of second primary tumors after lobectomy by means of video-assisted thoracoscopic surgery (VATS) versus thoracotomy for lung cancer. J. Thorac. Cardiovasc. Surg. 2011, 141, 59-64.

[3] Walker, W.S., Leaver, H.A. Immunologic and stress responses following video-assisted thoracic surgery and open pulmonary lobectomy in early stage lung cancer. Thorac. Surg. Clin. 2007, 17, 241-249, ix.

[4] Hu, J.K.;Zhou, Z.G.;Chen, Z.X.;Wang, L.L.;Yu, Y.Y.;Liu, J.;Zhang, B.;Li, L.;Shu, Y., Chen, J.P. Comparative evaluation of immune response after laparoscopical and open total mesorectal excisions with anal sphincter preservation in patients with rectal cancer. World J. Gastroenterol. 2003, 9, 2690-2694.

[5] Jackman, R.P.;Utter, G.H.;Muench, M.O.;Heitman, J.W.;Munz, M.M.;Jackman, R.W.;Biswas, H.H.;Rivers, R.M.;Tobler, L.H.;Busch, M.P., Norris, P.J. Distinct roles of trauma and transfusion in induction of immune modulation after injury. Transfusion. 2012, 52, 2533-2550.

[6] Kolseth, I.B.;Forland, D.T.;Risoe, P.K.;Flood-Kjeldsen, S.;Agren, J.;Reseland, J.E.;Lyngstadaas, S.P.;Johnson, E., Dahle, M.K. Human monocyte responses to lipopolysaccharide and 9-cis retinoic acid after laparoscopic surgery for colon cancer. Scand. J. Clin. Lab. Invest. 2012, 72, 593-601.

[7] Nosotti, M.;Rosso, L.;Mendogni, P.;Palleschi, A.;Tosi, D.;Bonara, P., Santambrogio, L. Leukocyte subsets dynamics following open pulmonary lobectomy for lung cancer: a prospective, observational study. Interact. Cardiovasc. Thorac. Surg. 2011, 13, 262-266.

[8] Hughes, D.;Otani, T.;Yang, P.;Newman, R.A.;Yantiss, R.K.;Altorki, N.K.;Port, J.L.;Yan, M.;Markowitz, S.D.;Mazumdar, M.;Tai, H.H.;Subbaramaiah, K., Dannenberg, A.J. NAD+dependent 15-hydroxyprostaglandin dehydrogenase regulates levels of bioactive lipids in nonsmall cell lung cancer. Cancer Prev. Res. (Phila). 2008, 1, 241-249.

[9] Rossi, A.;Cuzzocrea, S., Sautebin, L. Involvement of leukotriene pathway in the pathogenesis of ischemia-reperfusion injury and septic and non-septic shock. Curr. Vasc. Pharmacol. 2009, 7, 185-197.

[10] Sakamaki, F.;Ishizaka, A.;Handa, M.;Fujishima, S.;Urano, T.;Sayama, K.;Nakamura, H.;Kanazawa, M.;Kawashiro, T.;Katayama, M. Soluble form of P-selectin in plasma is elevated in acute lung injury. Am. J. Respir. Crit. Care Med. 1995, 151, 1821-1826.

[11] Stone, S.F., Brown, S.G. Mediators released during human anaphylaxis. Curr Allergy Asthma Rep. 2012, 12, 33-41.

[12] Utoh, J.;Yamamoto, T.;Utsunomiya, T.;Kambara, T.;Goto, H., Miyauchi, Y. Effect of surgery on neutrophil functions, superoxide and leukotriene production. Br. J. Surg. 1988, 75, 682-685.

[13] Van Ly, D.;Burgess, J.K.;Brock, T.G.;Lee, T.H.;Black, J.L., Oliver, B.G. Prostaglandins but not leukotrienes alter extracellular matrix protein deposition and cytokine release in primary human airway smooth muscle cells and fibroblasts. Am. J. Physiol. Lung Cell Mol. Physiol. 2012, 303, L239-250.

[14] Ytting, H.;Christensen, I.J.;Basse, L.;Lykke, J.;Thiel, S.;Jensenius, J.C., Nielsen, H.J. Influence of major surgery on the mannan-binding lectin pathway of innate immunity. Clin. Exp. Immunol. 2006, 144, 239-246.

[15] Li, Y.;Qiu, X.;Zhang, S.;Zhang, Q., Wang, E. Hypoxia induced CCR7 expression via HIF-1alpha and HIF-2alpha correlates with migration and invasion in lung cancer cells. Cancer Biol. Ther. 2009, 8, 322-330.

[16] Moungjaroen, J.;Nimmannit, U.;Callery, P.S.;Wang, L.;Azad, N.;Lipipun, V.;Chanvorachote, P., Rojanasakul, Y. Reactive oxygen species mediate caspase activation and apoptosis induced by lipoic acid in human lung epithelial cancer cells through Bcl-2 down-regulation. J. Pharmacol. Exp. Ther. 2006, 319, 1062-1069.

[17] Prabhu, N.;Raj, D.T.;Yamunagowri, Innocent, D.J.P. Pre and post operative immune status in patients enduring cardiac surgery. Int. J. Biol. Med. Res. 2010, 1, 253-258. 
[18] Shimoda, L.A., Semenza, G.L. HIF and the lung: role of hypoxia-inducible factors in pulmonary development and disease. Am. J. Respir. Crit. Care Med. 2011, 183, 152-156.

[19] Craig, S.R.;Leaver, H.A.;Yap, P.L.;Pugh, G.C., Walker, W.S. Acute phase responses following minimal access and conventional thoracic surgery. Eur. J. Cardiothorac. Surg. 2001, 20, 455-463.

[20] Leaver, H.A.;Craig, S.R.;Yap, P.L., Walker, W.S. Lymphocyte responses following open and minimally invasive thoracic surgery. Eur. J. Clin. Invest. 2000, 30, 230-238.

[21] Pavelkova, M.;Kubala, L.;Ciz, M.;Pavlik, P.;Wagner, R.;Slavik, J.;Ondrasek, J.;Cerny, J., Lojek, A. Blood phagocyte activation during open heart surgery with cardiopulmonary bypass. Physiological res. 2006, 55, 165.

[22] Hasenberg, M.;Stegemann-Koniszewski, S., Gunzer, M. Cellular immune reactions in the lung. Immunol. Rev. 2013, 251, 189-214.

[23] Kals, J.;Kampus, P.;Kals, M.;Pulges, A.;Teesalu, R.;Zilmer, K.;Kullisaar, T.;Salum, T.;Eha, J., Zilmer, M. Inflammation and oxidative stress are associated differently with endothelial function and arterial stiffness in healthy subjects and in patients with atherosclerosis. Scand. J. Clin. Lab. Invest. 2008, 68, 594-601.

[24] Rodiño-Janeiro, B.K.;Paradela-Dobarro, B.;Castiñeiras-Landeira, M.;Raposeiras-Roubín, S.;González-Juanatey, J., Álvarez, E. Current status of NADPH oxidase research in cardiovascular pharmacology. Vascular Health and Risk Management. 2013, 9, 401-428.

[25] Le Maux Chansac, B.;Misse, D.;Richon, C.;Vergnon, I.;Kubin, M.;Soria, J.C.;Moretta, A.; Chouaib, S., Mami-Chouaib, F. Potentiation of NK cell-mediated cytotoxicity in human lung adenocarcinoma: role of NKG2D-dependent pathway. Int. Immunol. 2008, 20, 801-810.

[26] Park, Y.;Kitahara, T.;Takagi, R., Kato, R. Does surgery for breast cancer induce angiogenesis and thus promote metastasis? Oncology. 2011, 81, 199-205.

[27] Schroder, K.;Zhang, M.;Benkhoff, S.;Mieth, A.;Pliquett, R.;Kosowski, J.;Kruse, C.;Luedike, P.;Michaelis, U.R.;Weissmann, N.;Dimmeler, S.;Shah, A.M., Brandes, R.P. Nox4 is a protective reactive oxygen species generating vascular NADPH oxidase. Circ. Res. 2012, 110, 1217-1225.

[28] van der Bilt, J.D., Borel Rinkes, I.H. Surgery and angiogenesis. Biochim. Biophys. Acta. 2004, $1654,95-104$

[29] Ng, C.S.;Wan, S.;Hui, C.W.;Wan, I.Y.;Lee, T.W.;Underwood, M.J., Yim, A.P. Video-assisted thoracic surgery lobectomy for lung cancer is associated with less immunochemokine disturbances than thoracotomy. Eur. J. Cardio-thoracic Surg. 2007, 31, 83-87.

[30] Sims, C.;Seigne, P.;Menconi, M.;Monarca, J.;Barlow, C.;Pettit, J., Puyana, J.C. Skeletal muscle acidosis correlates with the severity of blood volume loss during shock and resuscitation. $J$. Trauma. 2001, 51, 1137-1145; discussion 1145-1136.

[31] Tiziani, S.;Lodi, A.;Khanim, F.L.;Viant, M.R.;Bunce, C.M., Gunther, U.L. Metabolomic profiling of drug responses in acute myeloid leukaemia cell lines. PLoS One. 2009, 4, e4251.

[32] Rocha, C.M.;Carrola, J.;Barros, A.S.;Gil, A.M.;Goodfellow, B.J.;Carreira, I.M.;Bernardo, J.;Gomes, A.;Sousa, V.;Carvalho, L., Duarte, I.F. Metabolic signatures of lung cancer in biofluids: NMR-based metabonomics of blood plasma. J. Proteome Res. 2011, 10, 4314-4324.

[33] Wu, G.;Li, C.;Wang, L.;Mao, Y., Hong, Z. Minimally invasive procedures for evacuation of intracerebral hemorrhage reduces perihematomal glutamate content, blood-brain barrier permeability and brain edema in rabbits. Neurocrit. Care. 2011, 14, 118-126.

[34] Zetterling, M.;Hillered, L.;Samuelsson, C.;Karlsson, T.;Enblad, P., Ronne-Engstrom, E. Temporal patterns of interstitial pyruvate and amino acids after subarachnoid haemorrhage are related to the level of consciousness--a clinical microdialysis study. Acta Neurochir. (Wien). 2009, 151, 771-780; discussion 780.

[35] Shin, S.;Bai, S.J.;Rha, K.H.;So, Y., Oh, Y.J. The effects of combined epidural and general anesthesia on the autonomic nervous system and bioavailability of nitric oxide in patients undergoing laparoscopic pelvic surgery. Surg. Endosc. 2013, 27, 918-926.

[36] Pu, Q.;Ma, L.;Mei, J.D.;Zhu, Y.K.;Che, G.W.;Lin, Y.D.;Wu, Z.;Wang, Y.;Kou, Y.L.;Yang, J.J., Liu, L.X. [Immune functions of patients following lobectomy for lung cancers: a comparative study between video-assisted thoracoscopic surgery and posterolateral thoracotomy]. Sichuan Da Xие Xие Bao Yi Xue Ban. 2013, 44, 126-129.

[37] Camacho, M.;Rodríguez, C.;Guadall, A.;Alcolea, S.;Orriols, M.;Escudero, J.-R.;MartínezGonzález, J., Vila, L. Hypoxia upregulates PGI-synthase and increases PGI2 release in human vascular cells exposed to inflammatory stimuli. J. Lipid Res. 2011, 52, 720-731.

[38] Jung, Y.J.;Isaacs, J.S.;Lee, S.;Trepel, J., Neckers, L. IL-1beta-mediated up-regulation of HIF1alpha via an NFkappaB/COX-2 pathway identifies HIF-1 as a critical link between inflammation and oncogenesis. FASEB J. 2003, 17, 2115-2117. 
[39] Semple, S.J.;Smith, L.L.;McKune, A.J.;Hoyos, J.;Mokgethwa, B.;San Juan, A.F.;Lucia, A., Wadee, A.A. Serum concentrations of $\mathrm{C}$ reactive protein, alphal antitrypsin, and complement (C3, C4, C1 esterase inhibitor) before and during the Vuelta a Espana. Br. J. Sports Med. 2006, 40, 124-127.

[40] Weismann, D.;Hartvigsen, K.;Lauer, N.;Bennett, K.L.;Scholl, H.P.;Issa, P.C.;Cano, M.;Brandstätter, H.;Tsimikas, S., Skerka, C. Complement factor H binds malondialdehyde epitopes and protects from oxidative stress. Nature. 2011, 478, 76-81.

[41] Du Clos, T.W. Function of C-reactive protein. Ann Med. 2000, 32, 274-278.

[42] Christou, N.V., Meakins, J.L. Phagocytic and bactericidal functions of polymorphonuclear neutrophils from anergic surgical patients. Can. J. Surg. 1982, 25, 444-448.

[43] Circu, M.L., Aw, T.Y. Reactive oxygen species, cellular redox systems, and apoptosis. Free Radical Biol. and Med. 2010, 48, 749-762.

[44] O'Donnell, V.B., Murphy, R.C. New families of bioactive oxidized phospholipids generated by immune cells: identification and signaling actions. Blood. 2012, 120, 1985-1992.

[45] Higdon, A.;Diers, A.R.;Oh, J.Y.;Landar, A., Darley-Usmar, V.M. Cell signalling by reactive lipid species: new concepts and molecular mechanisms. Biochem. J. 2012, 442, 453-464.

[46] Wu, S.H.;Hang, L.W.;Yang, J.S.;Chen, H.Y.;Lin, H.Y.;Chiang, J.H.;Lu, C.C.;Yang, J.L.;Lai, T.Y.;Ko, Y.C., Chung, J.G. Curcumin induces apoptosis in human non-small cell lung cancer NCI-H460 cells through ER stress and caspase cascade- and mitochondria-dependent pathways. Anticancer Res. 2010, 30, 2125-2133.

[47] Davidson, J.;Rotondo, D.;Rizzo, M.T., Leaver, H.A. Therapeutic implications of disorders of cell death signalling: membranes, micro-environment, and eicosanoid and docosanoid metabolism. $\mathrm{Br}$. J. Pharmacol. 2012, 166, 1193-1210.

[48] Hong, S.H.;Kwak, J.H.;Paik, J.K.;Chae, J.S., Lee, J.H. Association of polymorphisms in FADS gene with age-related changes in serum phospholipid polyunsaturated fatty acids and oxidative stress markers in middle-aged nonobese men. Clin. Interventions in Aging. 2013, 8, 585-596.

[49] Carew, J.S.;Kelly, K.R., Nawrocki, S.T. Autophagy as a target for cancer therapy: new developments. Cancer Manag. Res. 2012, 4, 357-365.

[50] Lee, J.;Giordano, S., Zhang, J. Autophagy, mitochondria and oxidative stress: cross-talk and redox signalling. Biochem. J. 2012, 441, 523-540.

[51] Zoschke, D.C., Messner, R.P. Suppression of human lymphocyte mitogenesis mediated by phagocyte-released reactive oxygen species: comparative activities in normals and in chronic granulomatous disease. Clin. Immunol. Immunopathol. 1984, 32, 29-40.

[52] Gupta, A.;Zhuo, J.;Zha, J.;Reddy, S.;Olp, J., Pai, A. Effect of different intravenous iron preparations on lymphocyte intracellular reactive oxygen species generation and subpopulation survival. BMC Nephrol. 2010, 11, 16.

[53] Rohn, T.T.;Hinds, T.R., Vincenzi, F.F. Inhibition of Ca2+-pump ATPase and the Na+/K+-pump ATPase by iron-generated free radicals. Protection by 6,7-dimethyl-2,4-DI-1- pyrrolidinyl-7Hpyrrolo[2,3-d] pyrimidine sulfate (U-89843D), a potent, novel, antioxidant/free radical scavenger. Biochem. Pharmacol. 1996, 51, 471-476.

[54] Leaver, H.A.;Yap, P.L.;Rogers, P.;Wright, I.;Smith, G.;Williams, P.E.;France, A.J.;Craig, S.R.;Walker, W.S., Prescott, R.J. Peroxides in human leucocytes in acute septic shock: a preliminary study of acute phase changes and mortality. Eur. J. Clin. Invest. 1995, 25, 777-783.

[55] Aukrust, P.;Muller, F.;Nordoy, I.;Haug, C.J., Froland, S.S. Modulation of lymphocyte and monocyte activity after intravenous immunoglobulin administration in vivo. Clin. Exp. Immunol. 1997, 107, 50-56.

[56] Berzins, S.P.;Smyth, M.J., Baxter, A.G. Presumed guilty: natural killer T cell defects and human disease. Nat. Rev. Immunol. 2011, 11, 131-142.

[57] Michils, A.;Lambert, J.P.;Yernault, J.C.;Fabry, V.;Gossart, B., Duchateau, J. Fine tuning of epitopic dominance induced by lung cancer on the IgG response to bovine betalactoglobulin: towards a paraneoplastic immune marker. Cancer. 1996, 77, 657-664.

[58] Gonzalez-Quintela, A.;Lopez-Ben, S.;Perez, L.F.;Grana, B.;Varela, M.;Tome, S., Varo, E. Timecourse changes of serum immunoglobulins (IgA, IgG, IgM) after liver transplantation for alcoholic cirrhosis. Transpl. Immunol. 2003, 11, 73-77.

[59] Champault, G. [Has laparoscopic surgery reached its limits?]. J Chir (Paris). 1996, 133, 356-357.

[60] Ouyang, W.;Liao, W.;Luo, C.T.;Yin, N.;Huse, M.;Kim, M.V.;Peng, M.;Chan, P.;Ma, Q., Mo, Y. Novel Foxo1-dependent transcriptional programs control Treg cell function. Nature. 2012, 491, 554-559.

[61] Williams, M.S., Kwon, J. T cell receptor stimulation, reactive oxygen species, and cell signaling. Free Radical Biol. Med. 2004, 37, 1144-1151. 
[62] Remans, P.H.;Wijbrandts, C.A.;Sanders, M.E.;Toes, R.E.;Breedveld, F.C.;Tak, P.P.;van Laar, J.M., Reedquist, K.A. CTLA-4IG suppresses reactive oxygen species by preventing synovial adherent cell-induced inactivation of Rap1, a Ras family GTPASE mediator of oxidative stress in rheumatoid arthritis T cells. Arthritis Rheum. 2006, 54, 3135-3143.

[63] Gloire, G.;Legrand-Poels, S., Piette, J. NF-kappaB activation by reactive oxygen species: fifteen years later. Biochem. Pharmacol. 2006, 72, 1493-1505.

[64] Hamacher, J.;Stammberger, U.;Roux, J.;Kumar, S.;Yang, G.;Xiong, C.;Schmid, R.A.;Fakin, R.M.;Chakraborty, T.;Hossain, H.M.;Pittet, J.F.;Wendel, A.;Black, S.M., Lucas, R. The lectinlike domain of tumor necrosis factor improves lung function after rat lung transplantation-potential role for a reduction in reactive oxygen species generation. Crit. Care Med. 2010, 38, 871-878.

[65] Stein, S.J., Baldwin, A.S. NF-кB suppresses ROS levels in BCR-ABL\&plus; cells to prevent activation of JNK and cell death. Oncogene. 2011, 30, 4557-4566.

[66] Lofstedt, T.;Fredlund, E.;Holmquist-Mengelbier, L.;Pietras, A.;Ovenberger, M.;Poellinger, L., Pahlman, S. Hypoxia inducible factor-2alpha in cancer. Cell Cycle. 2007, 6, 919-926.

[67] Young, R.M., Simon, M.C. Untuning the tumor metabolic machine: HIF-alpha: pro- and antitumorigenic? Nat. Med. 2012, 18, 1024-1025.

[68] Song, X.;Liu, X.;Chi, W.;Liu, Y.;Wei, L.;Wang, X., Yu, J. Hypoxia-induced resistance to cisplatin and doxorubicin in non-small cell lung cancer is inhibited by silencing of HIF-1alpha gene. Cancer Chemother. Pharmacol. 2006, 58, 776-784.

[69] van Uden, P.;Kenneth, N.S., Rocha, S. Regulation of hypoxia-inducible factor-1alpha by NFkappaB. Biochem. J. 2008, 412, 477-484.

[70] Cook-Mills, J.M. VCAM-1 signals during lymphocyte migration: role of reactive oxygen species. Mol. Immunol. 2002, 39, 499-508.

[71] Cheng, Y.-J.;Chan, K.-C.;Chien, C.-T.;Sun, W.-Z., Lin, C.-J. Oxidative stress during 1-lung ventilation. J. Thoracic Cardiovas. Surg. 2006, 132, 513-518.

[72] Koch, C.G.;Li, L.;Sessler, D.I.;Figueroa, P.;Hoeltge, G.A.;Mihaljevic, T., Blackstone, E.H. Duration of red-cell storage and complications after cardiac surgery. N. Engl. J. Med. 2008, 358, 1229-1239.

[73] Ferru, E.;Giger, K.;Pantaleo, A.;Campanella, E.;Grey, J.;Ritchie, K.;Vono, R.;Turrini, F., Low, P.S. Regulation of membrane-cytoskeletal interactions by tyrosine phosphorylation of erythrocyte band 3. Blood. 2011, 117, 5998-6006.

[74] Steevels, T.A.;van Avondt, K.;Westerlaken, G.H.;Stalpers, F.;Walk, J.;Bont, L.;Coffer, P.J., Meyaard, L. Signal inhibitory receptor on leukocytes-1 (SIRL-1) negatively regulates the oxidative burst in human phagocytes. Eur. J. Immunol. 2013, 43, 1297-1308.

[75] Storz, P. Reactive oxygen species in tumor progression. Front Biosci. 2005, 10, 1881-1896.

[76] Hickey, M.J. Use of advanced imaging to generate novel insights in inflammation and adaptive immunity. Immunol.Cell Biol. 2013, 91, 261-262.

[77] Alaa RM, M.;Shibuya, K.;Fujiwara, T.;Wada, H.;Hoshino, H.;Yoshida, S.;Suzuki, M.;Hiroshima, K.;Nakatani, Y., Mohamed-Hussein, A.A. Risk of lung cancer in patients with preinvasive bronchial lesions followed by autofluorescence bronchoscopy and chest computed tomography. Lung Cancer. 2011, 72, 303-308. 